\title{
HGF, MET, and Matrix-Related Proteases in Hepatocellular Carcinoma, Fibrolamellar Variant, Cirrhotic and Normal Liver
}

\author{
Karen E. Schoedel, M.D., Valerie Zajac Tyner, B.S., Tae-Hyoung Kim, Ph.D.,
} George K. Michalopoulos, M.D., PhD., Wendy M. Mars, PhD.

Department of Pathology, University of Pittsburgh Medical Center, Pittsburgh, Pennsylvania

Fibrolamellar variant is an uncommon subcategory of hepatocellular carcinoma with a better prognostic outcome. Proteinases and growth factors that are involved in the remodeling of extracellular matrix may influence the behavior of cancers. To determine whether these factors contribute to the distinct etiologies of fibrolamellar hepatocellular carcinoma and traditional hepatocellular carcinoma, we assayed hepatocyte growth factor, the hepatocyte growth factor receptor, and two hepatocyte growth factor activators, hepatocyte growth factor activator and urokinasetype plasminogen activator, in hepatocellular carcinoma, fibrolamellar hepatocellular carcinoma, cirrhotic liver and normal liver. In addition, we examined the urokinase-type plasminogen activator receptor, the type 1 plasminogen activator inhibitor, plasmin, fibrinogen, and the type IV matrix metalloproteinases. Eighteen hepatocellular carcinomas and 11 fibrolamellar hepatocellular carcinomas were obtained as paraffin embedded sections from the University of Pittsburgh Department of Pathology. Frozen tissues from a subset of cases (9 hepatocellular carcinomas, 4 fibrolamellar hepatocellular carcinomas, 12 cirrhotic livers and 2 normal livers) were also available for analysis. Antibodies against urokinasetype plasminogen activator, urokinase-type plasminogen activator receptor, hepatocyte growth factor and hepatocyte growth factor receptor were used to analyze immunoperoxidase stained slides from the paraffin blocks. Western blot analyses using antibodies against hepatocyte growth factor, hepatocyte growth factor receptor, phosphotyrosine, hepatocyte growth factor activator, urokinase-type plasminogen activator receptor, urokinase-type plasminogen activator,

Copyright $(9) 2003$ by The United States and Canadian Academy of Pathology, Inc.

VOL. 16, NO. 1, P. 14, 2003 Printed in the U.S.A.

Date of acceptance: October 1, 2002.

Address reprint requests to: Wendy M. Mars, Ph.D., University of Pittsburgh, Department of Pathology, S 411 B Biomedical Science Tower, Pittsburgh, PA 15261; fax: 412-648-9846.

DOI: 10.1097/01.MP.0000043521.96995.DB plasminogen activator inhibitor-1, fibrinogen and plasmin were performed on membrane-enriched fractions from the frozen tissue, as was collagen $z y-$ mography for matrix metalloproteinase-2 and matrix metalloproteinase-9. The most notable findings are as follows: hepatocyte growth factor activator was only detected in malignant tissue but not cirrhotic liver or normal liver. Although hepatocyte growth factor was detected in most samples, it was significantly elevated in 5/9 hepatocellular carcinomas. Furthermore, 8/9 fibrolamellar hepatocellular carcinomas demonstrated hepatocyte growth factor receptor levels similar to normal, whereas 8/9 hepatocellular carcinomas and 11/12 cirrhotic livers exhibited either an increase or decrease. In contrast, active matrix metalloproteinase-2, which was absent in normal liver, was elevated in fibrolamellar hepatocellular carcinoma as compared to cirrhotic liver and conventional hepatocellular carcinoma. Surprisingly, 10/12 cirrhotic livers and 2/4 fibrolamellar hepatocellular carcinomas but only 1/9 hepatocellular carcinomas were enriched for plasmin. The combined data suggest that the hepatocyte growth factor and plasmin systems tend to be operative in hepatocellular carcinoma and cirrhotic liver, more than fibrolamellar hepatocellular carcinoma. Furthermore, matrix turnover appears to be a more prominent feature of fibrolamellar hepatocellular carcinoma. These findings provide insight into the behavioral differences between hepatocellular carcinoma and the fibrolamellar variant.

KEY WORDS: Fibrolamellar carcinoma, Hepatocellular carcinoma, Hepatocyte growth factor, MET, proteases.

Mod Pathol 2003;16(1):14-21

Hepatocellular carcinoma and the fibrolamellar variant differ in their respective clinical, gross, histologic and molecular features, which taken together may explain their different behaviors and clinical outcomes. Hepatocellular carcinoma is a 
relatively common neoplasm of the liver with well described clinical and pathologic characteristics. This neoplasm tends to arise in older males in the setting of cirrhosis and is often associated with other factors such as viral hepatitis and exposure to toxins. The tumor is commonly green due to its bile content, and although the neoplastic cells resemble hepatocytes cytologically, the tumor also demonstrates an array of architectural patterns. The prognosis is predominantly dependent on the stage of the neoplasm, however, because it is often discovered late in the course of disease, it is not often amenable to cure.

In contrast to conventional hepatocellular carcinoma, the fibrolamellar variant, also a malignant hepatic neoplasm, demonstrates different clinical, pathologic and behavioral features. Typically, fibrolamellar hepatocellular carcinoma arises in younger individuals with increased prevalence in females (approximately 1:1 male to female ratio) as compared to ordinary hepatocellular carcinoma that is found in a predominantly male population. The background liver is noncirrhotic and the prognosis is generally better than its conventional counterpart. The cytologic and histologic features are distinctive in that the tumor cells are embedded in a dense collagenous matrix. These neoplastic cells show oncocytic differentiation and resemble hepatocytes less closely than the tumor cells comprising ordinary hepatocellular carcinoma, although both tumors may share some similar nuclear features such as pseudo-inclusions and prominent nucleoli. Nevertheless, the matrices associated with these two neoplasms are vastly different. Hepatocellular carcinoma is associated with a prominent vascular pattern, but the fibrolamellar variant contains a dense fibrous matrix, histologically distinctive as compared to the desmoplastic reaction commonly associated with other carcinomas (1).

The clinical and histologic features of hepatocellular carcinoma and fibrolamellar hepatocellular carcinoma are well characterized; however, the complex cellular and extracellular matrix interactions that govern these tumors' behavior are not well understood. The dynamic processes of extracellular matrix turnover (for reviews see references $2,3,4)$ that contribute to the growth and development of normal as well as neoplastic tissues also play significant roles in these two neoplasms. The systems thought likely to be involved are the urokinase-type plasminogen activator/plasmin system (u-PA/plasmin), the hepatocyte growth factor/ MET receptor (HGF/MET) system, and the matrix metalloproteinase (MMP) system, all of which can act and interact to facilitate tumorigenesis. At present, these three systems only have been studied with respect to conventional hepatocellular carcinoma (5-20).
The $\mathrm{u}-\mathrm{PA} /$ plasmin system participates in the degradation of extracellular matrix by a complex set of processes involving cell surface receptors for u-PA which bind $\mathrm{u}-\mathrm{PA}$ and in turn facilitate plasminogen's conversion to plasmin, allowing for degradation of extracellular matrix proteins such as fibrin $(2,21)$. Plasmin and $\mathrm{u}$-PA also activate growth factors including transforming growth factor- $\beta$ and HGF $(22,23)$. The u-PA system is involved in cell migration, cell invasion, angiogenesis and is implicated in tumor metastasis. High levels of u-PA, $\mathrm{u}$-PAR and the type 1 plasminogen activator inhibitor (PAI-1) are associated with poor prognosis in many carcinomas and sarcomas $(7,21,24)$. Expression of $\mathrm{u}-\mathrm{PA}$ and $\mathrm{u}-\mathrm{PAR}$ mRNAs and overexpression of $\mathrm{u}-\mathrm{PA}, \mathrm{u}-\mathrm{PAR}$ and plasminogen activator inhibitor-1 (PAI-1) proteins have been documented in hepatocellular carcinoma (6-12).

HGF (also known as scatter factor) and its receptor, the tyrosine kinase MET, comprise a signaling pathway which can be mitogenic, motogenic, morphogenic, angiogenic and cytotoxic (for review see 25). Mesenchymal cells secrete the inactive form of HGF that must then be cleaved to form the active heterodimeric molecule (26). u-PA, tissue-type plasminogen activator, HGF activator (HGFa), blood coagulation factor XIIa and matriptase have all been reported to cleave single chain HGF to its active form $(23,27,28)$. The HGF/MET signaling pathway can induce u-PA and u-PAR expression $(6$, 29,30 ). u-PA in turn generates active HGF via cleavage of the inactive zymogen which can create a self-sustaining mechanism for tumorigenesis (23). Hepatocellular carcinomas co-express MET and HGF, which together are thought to represent an autocrine signaling mechanism for this tumor (5).

Besides the u-PA/plasmin system, MMPs comprise another group of proteins involved in the degradation of extracellular matrix and increased turnover of matrix. MMPs and tissue inhibitors of matrix metalloproteinases (TIMPs) are expressed in normal liver, cirrhotic liver and hepatocellular carcinoma. Hepatocellular carcinomas have been shown to contain MMPs 1,2 and 9 and stromelysins 1,2 and 3 (13). MMP-2 and MMP-9 have been localized to malignant hepatocytes (14) and are present in regenerating liver after hepatectomy (31).

This study was undertaken to investigate possible differences in the expression of these systems in fibrolamellar hepatocellular carcinoma with respect to hepatocellular carcinoma, cirrhotic liver, and normal liver. Our findings indicate that the HGF and plasmin systems are more dysfunctional in hepatocellular carcinoma, whereas matrix turnover is a more prominent feature of the fibrolamellar variant. 


\section{MATERIALS AND METHODS}

\section{Demographic Data}

The patients with hepatocellular carcinoma ranged in age from 22 to 71 years with a mean of 54.9 years and a male:female ratio of 2.0. With respect to fibrolamellar hepatocellular carcinoma, the patients ranged in age from 18 to 58 years with a mean of 32.5 years and a male:female ratio of 1.2.

\section{Immunoperoxidase Staining}

Eighteen hepatocellular carcinomas (15 moderately differentiated, 2 well differentiated and 1 poorly differentiated) and eleven fibrolamellar hepatocellular carcinomas were obtained as paraffin embedded material from the University of Pittsburgh Department of Pathology for immunoperoxidase studies using an indirect immunolabeling procedure with an Avidin-Biotin Complex method. The antibody sources and dilutions are listed in Table 1. Briefly, the blank slides were rehydrated and pretreated with a $30 \%$ solution of hydrogen peroxide in methanol for 15 minutes at room temperature. The slides then were washed in deionized water and placed in phosphate-buffered saline (PBS) plus $0.05 \%$ Tween 20 (Sigma Laboratories, Saint Louis, MO). The slides were blocked as follows: u-PA and u-PAR with $10 \%$ normal horse serum, and MET with $10 \%$ normal goat serum, for 20 minutes. The HGF slides were blocked with super block (ScyTek Laboratories, Logan, UT) for 2 minutes. The primary antibodies were incubated for one hour at room temperature. After washing in PBS, the secondary antibodies were incubated at room temperature for thirty minutes. The slides were washed again in PBS and incubated with Vectastain-Elite ABC (Vector Laboratories, Burlingame, CA) for 30 minutes at room temperature. The slides were developed with liquid 3,3'diaminobenzidine (Vector Laboratories) and counterstained with Shandon hematoxylin. Appropriate

TABLE 1. Antibody Sources and Dilutions

\begin{tabular}{llll}
\hline \multicolumn{1}{c}{ Antibody } & Primary & Secondary & \multicolumn{1}{c}{ Company (Primary) } \\
\hline Immunoperoxidase & & & \\
u-PA & $1: 100$ & $1: 200$ & American Diagnostica Inc. \\
u-PAR & $1: 25$ & $1: 200$ & American Diagnostica Inc. \\
HGF & $1: 70$ & $1: 200$ & R\&D \\
MET & $1: 75$ & $1: 200$ & Santa Cruz Biotech \\
Western Blot & & & \\
u-PA & $1: 500$ & $1: 2500$ & American Diagnostica Inc. \\
u-PAR & $1: 500$ & $1: 2500$ & American Diagnostica Inc. \\
pY & $1: 1000$ & NA & Transduction Laboratories \\
HGFa & $1: 400$ & $1: 2500$ & Santa Cruz \\
MET & $1: 1000$ & $1: 2500$ & Santa Cruz \\
HGF & $1: 500$ & $1: 2500$ & R\&D \\
PLM/PLG & $1: 1000$ & $1: 2500$ & Accurate \\
PAI-1 & $1: 1000$ & $1: 2500$ & American Diagnostica Inc. \\
FGN & $1: 1000$ & $1: 2500$ & ICN \\
\hline
\end{tabular}

positive controls were utilized as recommended by each antibody manufacturer. Cytoplasmic immunoreactivity of tumor cells, stromal cells, cirrhotic liver and normal liver was evaluated and scored semi-quantitatively $(0=$ no staining, $1+=$ weak staining, $2+=$ moderately strong staining, $3+=$ strong staining) Amount of tissue involved by the highest score was also estimated on each slide and recorded as less than $10 \%, 11-50 \%$ and greater than $50 \%$.

\section{Western Blot and Collagen Zymography}

Frozen tissue for use in western blot analysis and collagen zymography from a subset of these cases (9 hepatocellular carcinomas, 4 fibrolamellar hepatocellular carcinomas, 12 cirrhotic livers) was obtained from the University of Pittsburgh tissue bank. A portion of the sample was embedded in OCT media, a frozen section was performed and the resulting slide was stained with hematoxylin and eosin to verify the sample diagnosis before use in the study. Two normal liver samples were procured from autopsy material intended for hepatocyte transplant. MMP-2 and MMP-9 standards were obtained from the National Institutes of Health. For western blot and zymography analyses, membraneenriched fractions were prepared under detergent free conditions in $10 \mathrm{~mm}$ Tris- $\mathrm{HCl}, \mathrm{pH} 7.6$, with inhibitors $(10 \mathrm{ug} / \mathrm{mL}$ aprotinin, $5 \mathrm{ug} / \mathrm{mL}$ transEpoxysuccinyl-L-leucylamido-(4-guanidino) butane, $10 \mathrm{ug} / \mathrm{mL}$ pepstatin $\mathrm{A}, 1 \mathrm{mmol} / \mathrm{L}$ phenylmethylsulfonyl flouride, $50 \mathrm{umol} / \mathrm{L}$ 1,10-phenanthroline, $100 \mathrm{ug} / \mathrm{mL}$ sodium orthovanadate and $10 \mathrm{ug} / \mathrm{mL}$ leupeptin) by centrifuging the total cell lysate at $30,000 \mathrm{G}$ for 3 hours at 4 degrees $\mathrm{C}$ and separating the cytosol supernatant from the pellet. The pellets were resuspended in $1 \%$ sodium dodecyl sulfate (SDS) in $10 \mathrm{mmol} / \mathrm{L}$ Tris-HCL (pH7.6, also with inhibitors) and frozen at -80 degrees $\mathrm{C}$ until needed. The membrane-enriched fractions were mixed with SDS sample loading buffer, with or without 2-mercaptoethanol, and separated by electrophoresis through $8 \%$ or $10 \%$ SDSpolyacrylamide gels. Proteins were transferred to Immobilon-PVDF (Millipore, Bedford, MA). After transfer, even loading was confirmed by reversibly staining the membranes with Ponceau Red. The membranes were blocked with $5 \%$ nonfat dry milk in blotto solution $(20 \mathrm{mmol} / \mathrm{L}$ Tris- $\mathrm{HCl}(\mathrm{pH} 7.5)$, $150 \mathrm{mmol} / \mathrm{L} \mathrm{NaCl}, 0.1 \%$ Tween 20) for 1 to 2 hours at room temperature or overnight at 4 degrees $\mathrm{C}$. The membranes were incubated with primary antibody in 5\% nonfat dry milk blotto for 1 to 2 hours at room temperature, washed with $1 \%$ nonfat dry milk solution with blotto solution for 20 minutes at room temperature, 3 to 4 times, and then incubated with horseradish peroxidase-conjugated secondary 


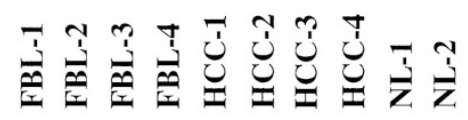

A.

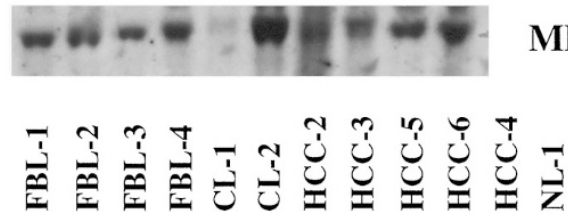

B.

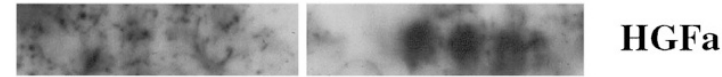

FIGURE 1. Western blots of MET (hepatocyte growth factor receptor) and HGFa (hepatocyte growth factor activator) from various liver tissues. Analyses were performed on membrane-enriched fractions from normal liver, cirrhotic liver, hepatocellular carcinoma and fibrolamellar hepatocellular carcinoma samples. An increase or decrease of MET is noted in HCC samples as compared to normal liver and fibrolamellar hepatocellular carcinoma samples. HGFa is present only in malignant tissues.

antibodies in the same buffer for 1 to 2 hours at room temperature. Subsequently, the membranes were washed for 20 minutes, 3 to 4 times, in blotto and incubated with ECL solution as recommended by the manufacturer (Amersham, Arlington Heights, IL). X-ray film (Dupont, Boston, MA) was used for detection of chemiluminescence.

To reutilize western blot filters, antibodies were stripped by shaking for one hour, 2 times, at 80 degrees centigrade in a solution containing $0.2 \mathrm{M}$ glycine, $\mathrm{pH} 2.5$, with $0.05 \%$ Tween- 20 and then rinsing with deionized water (32).

For collagen zymography, membrane-enriched fractions from the tumor samples were prepared in non-reducing sample buffer and separated by electrophoresis using a 9\% SDS polyacrylamide gel containing $400 \mathrm{ug} / \mathrm{mL}$ bovine skin gelatin. Gels were washed with $0.5 \%$ Triton X-100 for 30 minutes two to three times to remove the SDS. Subsequently, the gels were washed once with reaction buffer (50 $\mathrm{mmol} / \mathrm{L}$ Tris-HCL ( $\mathrm{pH}$ 7.5), $0.2 \mathrm{~mol} / \mathrm{L} \mathrm{NaCl}, 5$ $\mathrm{mmol} / \mathrm{L} \mathrm{CaCl}_{2}, 0.5 \mathrm{mg} / \mathrm{mL}$ Brij 35, $0.2 \mathrm{mg} / \mathrm{mL}$ $\mathrm{NaN}_{3}$ ) and then incubated in reaction buffer at 37 degrees $C$ for $36-38$ hours. The gels were stained with Coomassie Brilliant Blue and destained with a solution containing methanol/acetic acid/water (20:10:70). A clear zone indicated the presence of collagenase activity (31).

\section{RESULTS AND DISCUSSION}

In an effort to understand the different biologic behaviors of hepatocellular carcinoma, the fibrolamellar variant, cirrhotic liver and normal liver, three molecular systems were studied: HGF/c-MET, $\mathrm{u}-\mathrm{PA} /$ plasmin and matrix metalloproteinases. Potentially, the same mechanisms function in all tissues, but are emphasized differently in these respective neoplastic, preneoplastic and nonneoplastic livers.

\section{HGF/MET}

Western blot analyses could detect HGF in most samples including normals, but was elevated in 5 of 9 hepatocellular carcinomas. Immunoperoxidase expression of HGF was noted in the cytoplasm of the neoplastic cells of hepatocellular carcinoma and fibrolamellar hepatocellular carcinoma, as well as in the cytoplasm of the adjacent cirrhotic liver, non-cirrhotic liver, and stromal cells from the diseased organs (not shown).

Western blot analyses demonstrated abnormal quantities of MET (increased or decreased) as compared to normal liver in 8 of 9 hepatocellular carcinomas (Fig. 1). In 5 of 9 hepatocellular carcinomas the phosphoMET to MET ratio also varied from the norm. A decrease in MET (seen in 6 of 9 cases) was associated with a longer survival (Table 2). An increase or decrease of phosphoMET, MET and/or the ratio of phosphoMET to MET was also noted in 11 of 12 cirrhotic livers. Combined data from immunohistochemical staining (Fig. 2) and western blot analyses indicated that 8 of 9 fibrolamellar hepatocellular carcinomas displayed MET levels

TABLE 2. Correlation of Western Blot Results for MET and HGFa with Clinical Outcome

\begin{tabular}{|c|c|c|c|c|c|}
\hline ID & Grade & Outcome & Survival (mo) & MET & HGFa \\
\hline HCC-2 & 2 & Dead & 40 & Increase & Positive \\
\hline HCC -5 & 2 & Dead & 9 & Slight decrease & Positive \\
\hline HCC-7 & 3 & Dead & 6 & Same & Negative \\
\hline HCC-9 & 1 & Alive & 71 & Decrease & Positive \\
\hline HCC-4 & 2 & Alive & 57 & Decrease & Positive \\
\hline HCC-1 & 2 & Alive & 51 & Decrease & Negative \\
\hline HCC- 6 & 2 & Alive & 66 & Decrease & Positive \\
\hline HCC-8 & 2 & Alive & $36^{a}$ & Decrease & Negative \\
\hline HCC-3 & 2 & Alive & 56 & Decrease & Negative \\
\hline FBL-3 & & Dead & 41 & Same & Positive \\
\hline FBL-1 & & Dead & 32 & Same & Negative \\
\hline FBL-4 & & Dead & 11 & Same & Positive \\
\hline FBL-2 & & Alive & 84 & Same & Negative \\
\hline
\end{tabular}

${ }^{a}$ Current survival information is not available. 

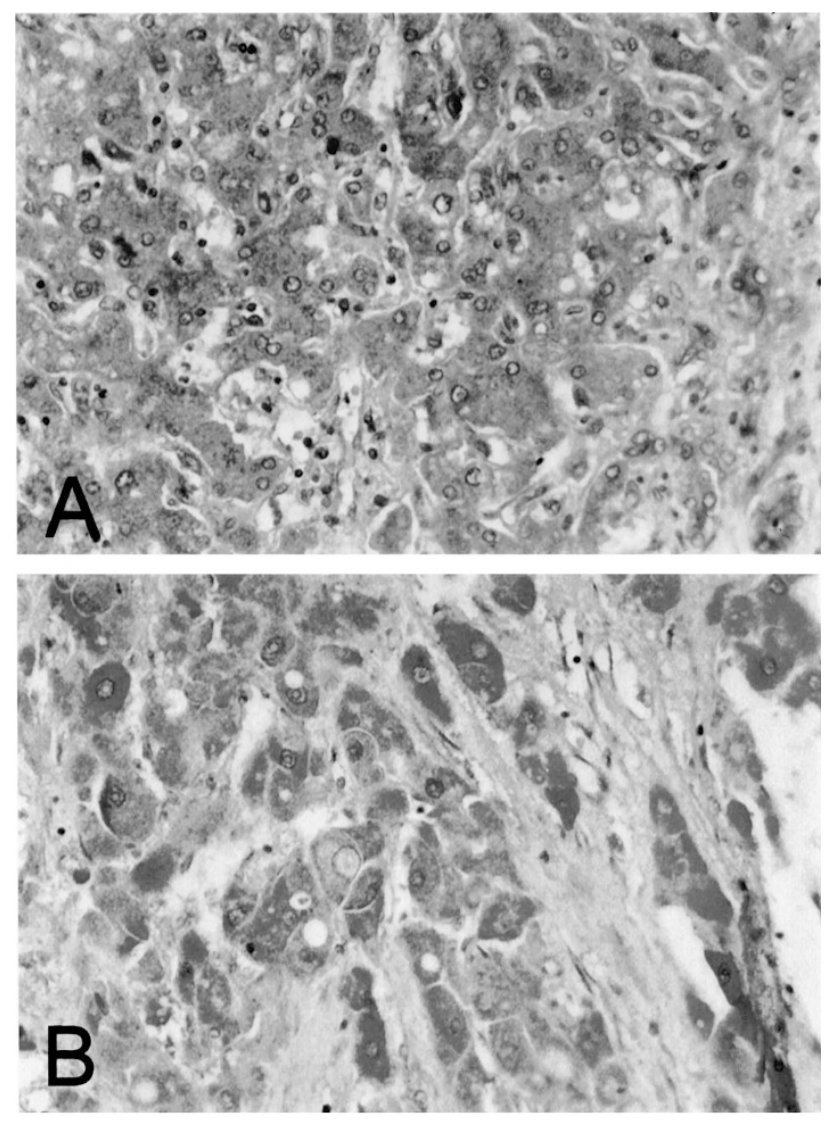

FIGURE 2. Cytoplasmic immunohistochemical reactivity for MET (hepatocyte growth factor receptor) is similar in (A) non-neoplastic liver and (B) adjacent fibrolamellar hepatocellular carcinoma (original magnification $200 \times$ )

similar to normal and only 1 of 4 fibrolamellar hepatocellular carcinomas showed a slight increase in phosphoMET by western blot analyses. Thus, our western blot findings with hepatocellular carcinoma are in general agreement with studies by other groups $(5,19)$ and show a significant difference from our findings with fibrolamellar hepatocellular carcinoma (see Figs. 1, 2 and Tables 2 and 3).

MET has been shown to be elevated in hepatocellular carcinoma as compared to non-tumor tis- sue and has been correlated with increased intrahepatic metastasis and decreased survival (19). Although both normal and hepatocellular carcinoma express MET (20), interaction between HGF and MET is necessary for tumorigenesis (25). It is important to note that the cirrhotic liver tissue used in this study was spatially located near neoplastic tissue. HGF is reportedly reduced or absent in cirrhotic liver and normal liver, but may be present in tissues adjacent to the tumor (5). Our finding of HGF by western blot in normal liver may be explained by the presence of HGF from bile ducts and stromal cells in whole liver samples (20) as normal hepatocytes do not express HGF (33).

Both HGF and MET were present in fibrolamellar hepatocellular carcinoma and immunoperoxidase studies showed the neoplastic cells stained positively for HGF. On the other hand, MET levels by western blot and immunostaining were similar to normal. The normal MET levels in the fibrolamellar variant suggest more controlled HGF/MET interactions in these tumors and indicate that other mechanisms are likely to be relevant to the behavior of fibrolamellar hepatocellular carcinoma. Previous literature with regard to fibrolamellar hepatocellular carcinoma is limited, but increased expression of fibronectin (34), the presence of tenascin in tumor stroma and abnormalities of cell adhesion molecule expression (e.g., cadherin and CD44) have been described in FBL (35).

\section{u-PA/plasmin and HGFa}

Both HGFa and u-PA can potentially interact with the HGF/MET pathway. In normal and neoplastic tissues, u-PA, either with or without its receptor u-PAR, or HGFa alone can activate the inactive form of $\operatorname{HGF}(23,27,36)$. Furthermore, HGF can induce expression of $\mathrm{u}-\mathrm{PA}$ and $\mathrm{u}-\mathrm{PAR}(6,30)$. When not interacting with HGF, u-PA is capable of generating plasmin from plasminogen, either with or without u-PAR.

Cytoplasmic immunoreactivity for $\mathrm{u}-\mathrm{PA}$ and $\mathrm{u}$-PAR was present in all of the neoplastic tissues

TABLE 3. Detection of HGF, MET and Matrix-Related Proteases by Western Blot and Collagen Zymography

\begin{tabular}{|c|c|c|c|c|c|c|c|c|c|c|c|c|}
\hline Tissue & HGFa & pMET & MET & Ratio $^{b}$ & HGF & $\mathrm{u}-\mathrm{PA}$ & u-PAR & PLM & PAI-1 & FDP & MMP2 & MMP9 \\
\hline NL & $0 / 2$ & $2 / 2^{a}$ & $2 / 2^{a}$ & - & $2 / 2^{a}$ & $0 / 2$ & $0 / 2$ & $0 / 2$ & $0 / 2$ & $0 / 2$ & $0 / 2$ & $2 / 2$ \\
\hline CL & $0 / 12$ & $6 / 12^{a}$ & $9 / 12^{a}$ & $7 / 12$ & $10 / 12^{a}$ & $7 / 12$ & $7 / 12$ & $10 / 12$ & $12 / 12$ & $1 / 12$ & $4 / 12$ & $10 / 12$ \\
\hline HCC & $6 / 9$ & $7 / 9^{a}$ & $8 / 9^{a}$ & $5 / 9$ & $9 / 9^{a}$ & $7 / 9$ & $6 / 9$ & $1 / 9$ & 9/9 & $6 / 9$ & $3 / 9$ & $2 / 9$ \\
\hline FBL & $2 / 4$ & $0 / 4^{a}$ & $1 / 9^{a}$ & $1 / 4$ & $2 / 4^{a}$ & $2 / 4$ & $0 / 4$ & $2 / 4$ & $4 / 4$ & $4 / 4$ & $4 / 4$ & $1 / 4$ \\
\hline
\end{tabular}

Data shown are a summary of Western blot (HGFa, pMET, MET, HGF, u-PA, u-PAR, PLM, PAI-1, FDP) and collagen zymography (MMP-2, MMP-9) analyses of normal liver, cirrhotic liver, hepatocellular carcinoma, and fibrolamellar hepatocellular carcinoma. The data for MET in fibrolamellar hepatocellular carcinoma samples combine the immunoperoxidase and Western blot data.

NL, normal liver; CL, cirrhotic liver; HCC, hepatocellular carcinoma; FBL, fibrolamellar variant of hepatocellular carcinoma.

${ }^{a}$ Small quantities of HGF, MET, and pMET were present in the normal autopsy materials. Therefore, table data for these proteins in the cirrhotic liver, hepatocellular carcinoma, and fibrolamellar hepatocellular carcinoma samples reflect a difference (either an increase or decrease) relative to the normal values.

${ }^{b}$ The ratio represents pMET/MET. Data for cirrhotic liver, hepatocellular carcinoma and the fibrolamellar variant reflect an increase or decrease of this ratio, relative to the ratio detected in normal liver. 
studied. A proteolytically active form of u-PA was detected by western blot analyses in 2 of 4 fibrolamellar hepatocellular carcinomas, 7 of 9 hepatocellular carcinomas, 7 of 12 cirrhotic livers and in none of the normal livers. 6 of 9 hepatocellular carcinomas and 7 of 12 cirrhotic livers expressed u-PAR by western blot; however, u-PAR was not detected in normal liver or in the fibrolamellar hepatocellular carcinoma samples (Table 3). Interestingly, western blot analysis for HGFa revealed that only malignant tissues (6/9 hepatocellular carcinomas, 2/4 fibrolamellar hepatocellular carcinomas and 0/12 cirrhotic livers tested, see Fig. 1) expressed the active form of this protein, suggesting that HGFa may be a relevant regulator of HGF, specific for the neoplastic state; however, no correlation was noted between quantity and survival rate (Table 2).

Western blot analysis was used to discriminate between plasminogen and plasmin. Detection of plasminogen was observed in all tissues. Surprisingly, only 1 of 9 hepatocellular carcinomas demonstrated plasmin although 10 of 12 cirrhotic livers tested positive and 2 of 4 fibrolamellar hepatocellular carcinoma samples contained plasmin (Fig. 3). Interestingly, PAI-1, generally considered to be an inhibitor of plasmin generation, was detectable in cirrhotic liver, hepatocellular carcinoma and the fibrolamellar variant but was more elevated in cirrhotic livers and fibrolamellar hepatocellular carcinomas than conventional hepatocellular carcinomas. Normal liver did not contain detectable plasmin or significant amounts of PAI-1 (Table 3).

Previous studies have demonstrated expression of u-PA mRNA in hepatocellular carcinoma and peritumoral cirrhotic liver $(8,9)$. u-PAR has also been documented in hepatocellular carcinoma $(8$,

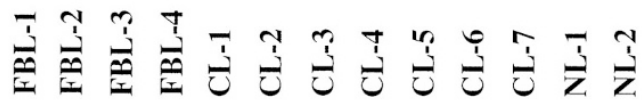
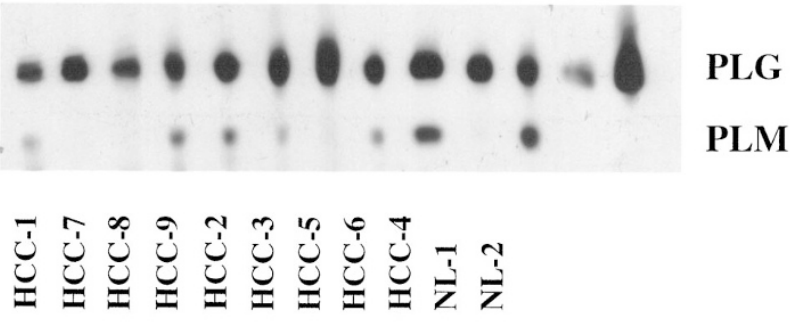

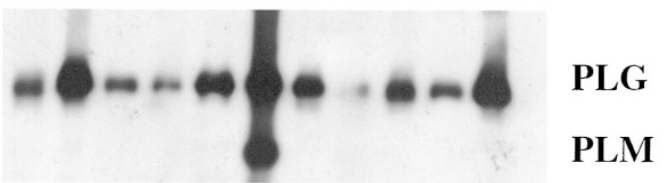

FIGURE 3. Western blots of PLG/PLM (plasminogen/plasmin) performed on membrane-enriched fractions of liver tissue. Plasminogen (PLG) is present in all samples and plasmin (PLM) is most apparent in cirrhotic liver samples.
$9,12)$. Our present study using immunohistochemical and western blot techniques corroborates the previous work. Immunoperoxidase studies showed expression of $\mathrm{u}-\mathrm{PA}$ and $\mathrm{u}-\mathrm{PAR}$ in the malignant hepatic tissues. Using western blot analyses, a band the size of active u-PA was identified in hepatocellular carcinoma, the fibrolamellar variant and cirrhotic liver, but not in normal liver. u-PAR was also present in hepatocellular carcinoma and cirrhotic liver but not in normal liver. Interestingly, although a normal size band for u-PAR was not detected in fibrolamellar hepatocellular carcinoma, a high molecular weight band of $155 \mathrm{kD}$ was present, suggesting that u-PAR may be present in these tissues in a complex with u-PA and PAI-1. Cells recycle functional complexes of $\mathrm{u}-\mathrm{PA} / \mathrm{u}-\mathrm{PAR}$ when PAI- 1 becomes bound to them (2).

The finding that plasmin is elevated in cirrhotic liver but rarely present in hepatocellular carcinomas is surprising. One possibility is that plasmin from the cirrhotic liver environment contributes to the neoplastic process of HCC by facilitating degradation of the extracellular matrix, allowing tumor growth at the junctions between cirrhotic liver and hepatocellular carcinoma. Similar situations have been reported for other types of tumors. An alternative hypothesis is that loss of plasmin by cirrhotic liver is an important step in the development of hepatocellular carcinoma. Studies using rodent liver have shown that plasmin is important for repair of injury in normal liver (37). Thus, the presence of plasmin in cirrhotic liver may be an attempt by the tissue to facilitate normal hepatocellular growth and the loss of plasmin may be an indication that the neoplastic process is dominant. The finding that 2 of 4 fibrolamellar hepatocellular carcinomas, a less aggressive type of HCC, were also positive for plasmin provides further support for this second hypothesis.

\section{Matrix Metalloproteinases and Fibrinogen}

In addition to the $\mathrm{u}-\mathrm{PA} /$ plasmin system, matrix metalloproteinases (MMPs) are involved in proteolytic degradation of the extracellular matrix and play important roles in tumor invasion and metastasis $(3,4,38)$. The MMP system also interacts with the HGF/MET and u-PA/plasmin systems. ProMMP-2 is activated by plasmin and is inhibited by plasmin inhibitors and anti-u-PA antibody (39). Collagenases can also increase the mitogenic effect of HGF (40). Expression of specific MMPs and their inhibitors has been found in normal liver, cirrhotic liver, and hepatocellular carcinoma (13, 14). MMP-9 and MMP-2 have been implicated in the invasive behavior of conventional hepatocellular carcinoma $(15,16,17)$. 
We performed collagen zymography for active MMP-2 and MMP-9. Active (62 kDa) MMP-2 was most elevated in fibrolamellar hepatocellular carcinoma samples as compared to hepatocellular carcinoma and cirrhotic liver and was absent from normal liver. MMP-9 was highest in normal liver and cirrhotic liver as compared to the malignant tissues (Fig. 4). The results with the normal liver samples are the same as previously reported; however, the results with the cirrhotic livers and hepatocellular carcinomas differ in that the predominant form we detected was $62 \mathrm{kDa}$, rather than 66 $\mathrm{kDa}$ (17). This probably reflects our use of membrane-enriched fractions as opposed to whole liver extracts. When matrix was examined in the tissues, western blot analysis for fibrinogen/fibrin showed that all fibrolamellar hepatocellular carcinoma samples had low molecular weight fragments (lmwFN) as compared to 6 of 9 hepatocellular carcinomas and $1 / 12$ cirrhotic livers (Table 3 ). In the hepatocellular carcinomas and cirrhotic livers, the findings of lmwFN in the absence of detectable plasmin (hepatocellular carcinomas) and plasmin without lmwFN (cirrhotic livers) was surprising. These fragments are generally thought to correspond to plasmin-induced fibrin degradation products (41). Nevertheless, combined, the data indicate that matrix turnover is a more prevalent feature in fibrolamellar hepatocellular carcinoma and the results support different avenues of extracellular matrix degradation with regard to conventional hepatocellular carcinoma and the fibrolamellar variant.

In summary, hepatocellular carcinoma and the fibrolamellar variant appear to use different pathways to achieve invasion and metastasis. In fibrolamellar hepatocellular carcinoma, the u-PA/plasmin system and the MMP system interact to facilitate extracellular matrix turnover. By contrast, the HGF/MET system appears more relevant in hepatocellular carcinoma as opposed to the fibrolamellar variant. Cirrhotic liver appears to represent a condition with some phenotypic similarities to

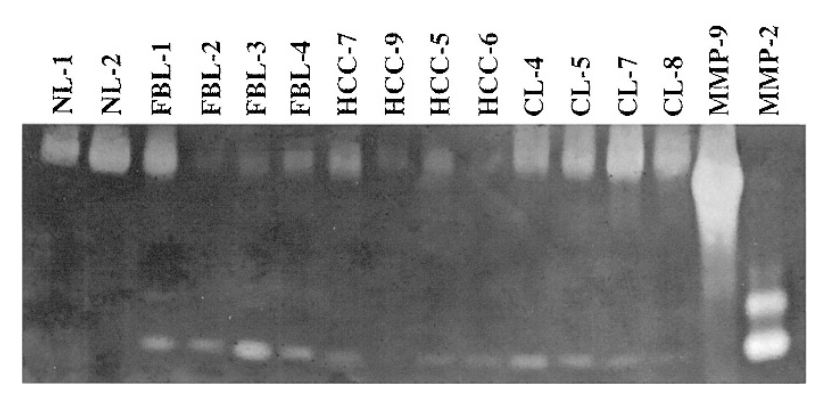

FIGURE 4. Collagen zymography for active matrix metalloproteinase2 (MMP-2) and matrix metalloproteinase-9 (MMP-9). The data show that the $62 \mathrm{kDa}$ form of MMP-2 is most elevated in fibrolamellar hepatocellular carcinoma samples as compared to conventional hepatocellular carcinoma, cirrhotic liver and normal liver. hepatocellular carcinoma, although surprisingly, loss of plasmin seems to precede the malignant process. Finally, HGFa is present specifically in cancerous tissues, suggesting a correlation between this serine protease and hepatocellular malignancies.

Acknowledgments: The authors thank Kari Nejak and Kim Stanis-Fuhrer for their technical assistance. This work has been supported by the UPMC Department of Pathology.

\section{REFERENCES}

1. Demay R. Liver. In: The art and science of cytopathology. Hong Kong: Aspiration Cytology, ASCP; 1996. pp. 1026-31.

2. Blasi F. Molecular mechanisms of protease-mediated tumor invasiveness. J Surg Oncol Suppl 1993;3:21-3.

3. Murphy G, Docherty AJ. The matrix metalloproteinases and their inhibitors. Am J Respir Cell Mol Biol 1992;7:120-5.

4. Stetler-Stevenson WG, Liotta LA, Kleiner DE. Extracellular matrix 6: role of matrix metalloproteinases in tumor invasion and metastasis. FASEB J 1993;7:1434-41.

5. Ljubimova JY, Petrovic LM, Wilson SE, Geller SA, Demetriou AA. Expression of HGF, its receptor c-met, c-myc and albumin in cirrhotic and neoplastic human liver tissue. J Histochem Cytochem 1997;45:79-87.

6. Monvoisin A, Neaud V, De Ledinghen V, Dubuisson L, Balabaud C, Bioulac-Sage P, et al. Direct evidence that hepatocyte growth factor-induced invasion of hepatocellular carcinoma cells is mediated by urokinase. J Hepatol 1999;30: 511-8.

7. Itoh T, Hayashi Y, Kanamaru T, Morita Y, Suzuki S, Wang W, et al. Clinical significance of urokinase-type plasminogen activator activity in hepatocellular carcinoma. J Gastoenterol Hepatol 2000;15:422-30.

8. De Petro G, Tavian D, Copeta A, Portolani N, Giulini SM, Barlati S. Expression of urokinase-type plasminogen activator (u-PA), u-PA receptor, and tissue-type PA messengerRNAs in human hepatocellular carcinoma. Cancer Res 1998; 58:2234-9.

9. Dubuisson L, Monvoisin A, Nielsen BS, Le Bail B, BioulacSage P, Rosenbaum J. Expression and cellular localization of the urokinase-type plasminogen activator and its receptor in human hepatocellular carcinoma. J Pathol 2000;190:190-5.

10. Zheng Q, Tang ZY, Xue Q, Shi DR, Song HY, Tang HB. Invasion and metastasis of hepatocellular carcinoma in relation to urokinase-type plasminogen activator, its receptor and inhibitor. J Cancer Res Clin Oncol 2000;126:641-6.

11. Zhou L, Hayashi Y, Itoh T, Wang W, Rui J, Itoh H. Expression of urokinase-type plasminogen activator, urokinase-type plasminogen activator receptor, and plasminogen activator inhibitor-1 and-2 in hepatocellular carcinoma. Pathol Int 2000;50:392-7.

12. Morita Y, Hayashi Y, Wang Y, Kanamaru T, Suzuki S, Kawasaki K, et al. Expression of urokinase-type plasminogen activator receptor in hepatocellular carcinoma. Hepatology 1997;25:856-61.

13. Lichtinghagen R, Helmbrecht T, Arndt B, Boeker KH. Expression pattern of matrix metalloproteinases in human liver. Eur J Clin Chem Clin Biochem 1995;33:65-71.

14. Arii S, Mise M, Harada T, Furutani M, Ishigami S, Niwano M, et al. Overexpression of matrix metalloproteinase 9 gene in hepatocellular carcinoma with invasive potential. Hepatology 1996;24:316-22. 
15. Ogata R, Torimura T, Kin M, Ueno T, Tateishi Y, Kuromatsu $\mathrm{R}$, et al. Increased expression of membrane type-1 matrix metalloproteinase and matrix metalloproteinase- 2 with tumor dedifferentiation in hepatocellular carcinomas. Hum Pathol 1999;30:443-50.

16. Ashida K, Nakatsukasa H, Higashi T, Ohguchi S, Hino N, Nouso K, et al. Cellular distribution of 92-kd type IV collagenase/gelatinase B in human hepatocellular carcinoma. Am J Pathol 1996;149:1803-11.

17. Theret N, Musso O, Turlin B, Lotrian D, Bioulac-Sage P, Campion JP, et al. Increased extracellular matrix remodeling is associated with tumor progression in human hepatocellular carcinomas. Hepatology 2001;34:82-8.

18. Yamaguchi K, Nalesnik MA, Michalopoulos GK. Hepatocyte growth factor mRNA in human liver cirrhosis as evidenced by in situ hybridization. Scand J Gastroenterol 1996;31:921-7.

19. Ueki T, Fujimoto J, Suzuki T, Yamamoto H, Okamoto E. Expression of hepatocyte growth factor and its receptor, the c-met proto-oncogene, in hepatocellular carcinoma. Hepatology 1997;25:619-23.

20. Chen Q, Seol DW, Carr B, Zarnegar R. Co-expression and regulation of Met and Ron proto-oncogenes in human hepatocellular carcinoma tissues and cell lines. Hepatology 1997;26:59-66.

21. Andreasen PA, Kjoller L, Christensen L, Duffy MJ. The urokinase-type plasminogen activator system in cancer metastasis: a review. Int J Cancer 1997;72:1-22.

22. Kojima S, Hayashi S, Shimokado K, Suzuki Y, Shimada J, Crippa MP, et al. Transcriptional activation of urokinase by the Kruppel-like factor Zf9/COPEB activates latent TGF-B1 in vascular endothelial cells. Blood 2000;95:1309-16.

23. Mars WM, Zarnegar R, Michalopoulos GK. Activation of hepatocyte growth factor by the plasminogen activators $\mathrm{u}-\mathrm{PA}$ and t-PA. Am J Pathol 1993;143:949-58.

24. Fazioli F, Blasi F. Urokinase-type plasminogen activator and its receptor: new targets for anti-metastatic therapy? Trends Pharmacol Sci 1994;15:25-9.

25. Jeffers M, Rong S, Vande Woude GF. Hepatocyte growth factor/scatter factor-MET signaling in tumorigenicity and invasion/metastasis. J Mol Med 1996;74:505-13.

26. Lokker NA, Mark MR, Luis EA, Bennett GL, Robbins KA, Baker JB, et al. Structure-function analysis of hepatocyte growth factor: identification of variants that lack mitogenic activity yet retain high affinity receptor binding. EMBO J 1992;11:2503-10.

27. Shimomura T, Miyazawa K, Komiyama Y, Hiraoka H, Naka D, Morimoto Y, et al. Activation of hepatocyte growth factor by two homologous proteases, blood coagulation factor XIIa and hepatocyte growth factor activator. Eur J Biochem 1995; 229:257-61.

28. Lee SL, Dickson RB, Lin CY. Activation of hepatocyte growth factor and urokinase/plasminogen activator by matriptase, an epithelial membrane serine protease. J Biol Chem 2000; 275:36720-5.

29. Ried S, Jaeger C, Jeffers M, Vande Woude GF, Graeff H, Schmitt M, et al. Activation mechanisms of the urokinasetype plasminogen activator promoter by hepatocyte growth factor/scatter factor. J Biol Chem 1999;274:16377-86.

30. Pepper MS, Matsumoto K, Nakamura T, Orci L, Montesano R. Hepatocyte growth factor increases urokinase-type plasminogen activator ( $\mathrm{u}-\mathrm{PA})$ and $\mathrm{u}-\mathrm{PA}$ receptor expression in Madin-Darby canine kidney epithelial cells. J Biol Chem 1992;267:20493-6.

31. Kim TH, Mars WM, Stolz DB, Michalopoulos GK. Expression and activation of pro-MMP-2 and pro-MMP-9 during rat liver regeneration. Hepatology 2000;31:75-82.

32. Stolz DB, Mars WM, Petersen BE, Kim T-H, Michalopoulos GK. Growth factor signal transduction after two-thirds partial hepatectomy in the rat. Cancer Res 1999;59:3954-60.

33. Wolf HK, Zarnegar R, Michalopoulos GK. Localization of hepatocyte growth factor in human and rat tissues: an immunohistochemical study. Hepatology 1991;14:488-94.

34. Jagirdar J, Ishak KG, Columbo M, Brambilla C, Paronetto F. Fibronectin patterns in hepatocellular carcinoma and its clinical significance. Cancer 1985;56:1643-8.

35. Scoazec JY, Flejou JF, D'Errico A, Fiorentino M, Zamparelli A, Bringuier $\mathrm{AF}$, et al. Fibrolamellar carcinoma of the liver: composition of the extracellular matrix and expression of cell-matrix and cell-cell adhesion molecules. Hepatology 1996;24:1128-36.

36. Mars WM, Kim TH, Stolz DB, Liu ML, Michalopoulos GK. Presence of urokinase in serum-free primary rat hepatocyte cultures and its role in activating hepatocyte growth factor. Cancer Res 1996;56:2837-43.

37. Bezerra JA, Bugge TH, Melin-Aldana H, Sabla G, Kombrinck KW, Witte DP, et al. Plasminogen deficiency leads to impaired remodeling after a toxic injury to the liver. Proc Natl Acad Sci USA 1999;96:15143-8.

38. Cottam DW, Rees RC. Regulation of matrix metalloproteinases: their role in tumor invasion and metastasis. Int J Oncol 1993;2:861-72.

39. Baramova EN, Bajou K, Remacle A, L'Hoir C, Krell HW, Weidle UH, et al. Involvement of PA/plasmin system in the processing of pro-MMP-9 and in the second step of proMMP-2 activation. FEBS Lett 1997;405:157-62.

40. Liu ML, Mars WM, Zarnegar R, Michalopoulos GK. Collagenase pretreatment and the mitogenic effects of hepatocyte growth factor and transforming growth factor-alpha in adult rat liver. Hepatology 1994;19:1521-7.

41. Kim TH, Mars WM, Stolz DB, Petersen BE, Michalopoulos GK. Extracellular matrix remodeling at the early stages of liver regeneration in the rat. Hepatology 1997;26:896-904. 